\title{
Three-Dimensional Aeroacoustic Numerical Simulation of Flow Induced Noise of Mufflers
}

\author{
Yan Yang ${ }^{1, \star}$ and Hongling Sun $^{2}$ \\ 1 LHD, Institute of Mechanics, Chinese Academy of Sciences, \\ No. 15 Beisihuanxi Road, Beijing 100190, P.R. China \\ yangy@ustc.edu \\ 2 Key Laboratory of Noise and Vibration Research, Institute of Acoustics, \\ Chinese Academy of Sciences, No. 21 Beisihuanxi Road, Beijing 100190, P.R. China \\ hlsun@mail.ioa.ac.cn
}

\begin{abstract}
Mufflers are widely applied in industrial flow duct systems or internal combustion engines, to reduce the amount of noise carried by the upstream flow. Although the flow in the duct of the muffler is commonly unsteady, complex and turbulent, which generates noise by itself. The flow noise should be considered for design and optimization of the muffler. By means of a three-dimensional numerical simulation integrated CFD (computational fluid dynamics) and CAA (computational aeroacoustics), the paper investigated two typical mufflers. The first one has an expanded chamber in the duct, and the second one has the same chamber but whilst has a perforated wall between the duct and the chamber. The nonlinear acoustic solver is implemented to model noise generation and transmission from an initial statistically-steady turbulent flow, which provided by RANS (Reynolds-averaged Navier-Stokes) simulation, and to simulate the noise in near field. The radiated far-field noise of the mufflers was predicted by FW-H (Ffowcs Williams-Hawking) acoustic analogy. The mechanisms of the vortex and sound generation were revealed, and results indicate that the perforated tube muffler has much lower flow induced noise level. The solver of the numerical simulation has been parallelized with MPI, and run on a HPC cluster, due to the large computation cost.
\end{abstract}

Keywords: computational fluid dynamics (CFD), computational aeroacoustics (CAA), flow induced noise, muffler.

\section{Introduction}

Mufflers (or silencers) are commonly used as silencing elements in HVAC ducts, automotive exhaust systems or other internal fluid machineries, to attenuate the noise emitted by upstream sound sources. The acoustic attenuation mechanisms in the absence of flow medium have been quite well understood. However, flow in the duct and chamber of muffler is commonly unsteady, complex and turbulent, which generates noise by itself. The flow induced noise should be carefully

\footnotetext{
* Corresponding author.
} 
considered for design and optimization of the muffler, especially at high speed [1-6].

With development of computational fluid dynamics (CFD) and computational aeroacoustics (CAA), numerical simulation should be able to applied to predict the flow excited noise. The methods of CAA can be classified in two basic approaches, the first one is directional approach, also called as directional noise computation (DNC) and the second is hybrid approach [7, 8]. Because the acoustic perturbation is much smaller than the flow dynamic quantities, the directional noise computation often costs a lot and the numerical error should overwhelm the acoustic quantities. In order to accurately simulate sound in the flow with acceptable computational amount, hybrid approach is commonly adopted, that is of two-step, i) acoustic source computation and ii) sound propagation computation, where different methods are used in corresponding domains and integrated through data transmission. The first step should be accomplished by directional computational fluid dynamic simulation, through RANS/LES/DNS approach, where the flow perturbation and acoustic source are computed or modeled. The second step, in general, consists of sound computation in near field and far field, of which the former is to solve the partial derivative equation of acoustic perturbation and the later is to solve the acoustic analogy equation or other similar equations. In present work, the numerical method is based on the NLAS (nonlinear acoustic solver) approach, derived by Batten et al [9, 10]. NLAS provides a more sophisticated sub-grid treatment that allows the extraction of acoustic sources from the temporal variation within the (modeled) sub-grid structures. The main advantage of this approach is that the noise in the near field can be simulated with less computation cost comparing to the directional noise computation approaches with traditional LES, hybrid RANS/LES, because the grid requirements is relaxed in the near-wall region. Furthermore, it has the important advantage of being able to account for both for broadband, turbulence-related noise and discrete tones arising from coherent structures or resonance, which can not be neglected in present study on the flow noise issues of mufflers.

Two typical kinds of mufflers were studied in this paper, one of which is constructed by a expanded chamber in the duct, and another with a perforated wall between the chamber and the duct. With the qualitative and quantitative comparison with experimental measurement shows that the simulation has credible results. The results helps to understand the mechanisms and control of the flow induced noise in the typical mufflers, which is great useful for the muffler design.

\section{Numerical Methods}

In present work, the hybrid approach of CAA, NLAS was implemented. The strategy can be described as the four steps as below. Firstly, the CFD simulation is performed to obtain the averaged flow field, with a conventional RANS method. Followed that, the noise source fluctuations are generated by the synthetic reconstruction of turbulence. Then, the acoustic perturbation equations, called nonlinear disturbance equation (NLDE), are solved though the NLAS. 
Finally, the far-field sound pressure is computed by the FW-H acoustic analogy. Following subsections present the details of the methods.

In order to compute the broad-band sound noise, because of the large computational cost, the parallel computation is implemented in the RANS step, and in the NLAS step. The domain partition was done with the tool package of metis [11].

\subsection{Noise Source Computation}

Firstly, the standard RANS with $k-\epsilon$ model is chosen to model the turbulence in the study. The governing equations of the viscous compressible Navier-Stokes equations of perfect gas are given with index notation as:

$$
\frac{\partial Q}{\partial t}+\frac{\partial F_{i}}{\partial x_{i}}-\frac{\partial G_{i}}{\partial x_{i}}=\dot{S}
$$

where $Q$ is the dependent variable vector; $F_{i}$ is the inviscid flux vector; $G_{i}$ is the viscous flux vector; and $\dot{S}$ is the source term vector, equal to zero here. They are given as:

$$
Q=\left(\begin{array}{c}
\rho \\
\rho u_{j} \\
e
\end{array}\right), F_{i}=\left(\begin{array}{c}
\rho u_{i} \\
\rho u_{i} u_{j}+p \delta_{i j} \\
u_{i}(e+p)
\end{array}\right), G_{i}=\left(\begin{array}{c}
0 \\
\tau_{i j} \\
-\theta_{i}+u_{k} \tau_{k i}
\end{array}\right),
$$

where $\rho$ is the density, $e$ is the total energy, $p$ is the pressure, $u_{i}$ is the velocity, $\theta_{i}$ is the heat flux, and $\tau_{i j}$ is the viscous stress tensor. With linear turbulence models, the dynamic viscosity coefficient $\mu$ in the constitutive relationship becomes to $\mu+\mu_{t}$ and the thermal conductivity $k$ becomes to $k+k_{t}$.

The two-equation nonlinear (cubic) $k-\epsilon$ model is used to obtain Reynolds stresses from the modeled eddy viscosity $\left(\mu_{t}\right)$ and the available mean-strain tensor. The model equations are written as:

$$
\begin{gathered}
\frac{\partial}{\partial t}(\bar{\rho} \tilde{k})+\frac{\partial}{\partial x_{i}}\left(\bar{\rho} \tilde{u}_{i} \tilde{k}\right)=\frac{\partial}{\partial x_{i}}\left[\left(\mu+\frac{\mu_{t}}{\sigma_{k}}\right) \frac{\partial \tilde{k}}{\partial x_{j}}\right]+P_{k}-\bar{\rho} \tilde{\epsilon} \\
\frac{\partial}{\partial t}(\bar{\rho} \tilde{\epsilon})+\frac{\partial}{\partial x_{i}}\left(\bar{\rho} \tilde{u}_{i} \tilde{\epsilon}\right)=\frac{\partial}{\partial x_{i}}\left[\left(\mu+\frac{\mu_{t}}{\sigma_{\epsilon}}\right) \frac{\partial \tilde{\epsilon}}{\partial x_{j}}\right]+\left(C_{\epsilon 1} P_{k}-C_{\epsilon 1} \bar{\rho} \tilde{\epsilon}+E\right) T_{t}^{-1} .
\end{gathered}
$$

Because the flow in the mufflers is entirely low-speed $(M=0.03)$, the preconditioning approach [12] is adopted to overcome the numerical issues encountered by the standard algorithm of compressible flow equations.

Secondly, the synthetic reconstruction of turbulence is used to generate the a full-spectrum noise source from the set of RANS turbulence statistics. This step is important for use with acoustic wave propagation equations solver, either numerical or analytic methods. Details of the synthetic method adopted in the study can be found in [10]. 


\subsection{Noise Propagation Computation}

The hybrid CAA approach NLAS, was developed by Batten et al [9, 10], advanced from the derivation of Morris et al [13]. The governing equations are referred to as nonlinear disturbance equations (NLDE), derived from the origninal Navier-Stokes equations (1), by rearranging for fluctuation and mean quantities, written as:

$$
\frac{\partial Q^{\prime}}{\partial t}+\frac{\partial F_{i}^{\prime}}{\partial x_{i}}-\frac{\partial G_{i}^{\prime}}{\partial x_{i}}=-\frac{\partial \bar{Q}}{\partial t}-\frac{\partial \bar{F}_{i}}{\partial x_{i}}+\frac{\partial \bar{G}_{i}}{\partial x_{i}},
$$

where

$$
\begin{gathered}
\bar{Q}=\left(\begin{array}{c}
\bar{\rho} \\
\overline{\rho u_{j}} \\
\bar{e}
\end{array}\right), \bar{F}_{i}=\left(\begin{array}{c}
\bar{\rho}_{i} \\
\overline{\rho u_{i}} \bar{u}_{j}+\bar{p} \delta_{i j} \\
\bar{u}(\bar{e}+\bar{p})
\end{array}\right), \bar{G}_{i}=\left(\begin{array}{c}
0 \\
\bar{\tau}_{i j} \\
-\bar{\theta}_{i}+\bar{u}_{k} \bar{\tau}_{k i}
\end{array}\right), \\
Q^{\prime}=\left(\begin{array}{c}
\rho^{\prime} \\
\bar{\rho} u_{j}^{\prime}+\rho^{\prime} u_{j}^{\prime} \\
e^{\prime}
\end{array}\right), G_{i}^{\prime}=\left(\begin{array}{c}
0 \\
\tau_{i j}^{\prime} \\
-\theta_{i}^{\prime}+u_{k}^{\prime} \bar{\tau}_{k i}+\bar{u}_{k} \tau_{k i}^{\prime}
\end{array}\right), \\
F_{i}^{\prime}=\left(\begin{array}{c}
\rho^{\prime} u_{i}^{\prime} \\
\rho^{\prime} \bar{u}_{i} \bar{u}_{j}+\bar{\rho} u_{i}^{\prime}+\rho^{\prime} \bar{u}_{i} \\
\bar{\rho} u_{i}^{\prime}+\bar{\rho} u_{i}^{\prime} \bar{u}_{j}+p^{\prime} \delta_{i j} \\
u_{i}^{\prime}+\bar{u}_{i}\left(e^{\prime}+p^{\prime}\right)
\end{array}\right)+\left(\begin{array}{c}
\bar{\rho} u_{i}^{\prime} u_{j}^{\prime}+\rho^{\prime} u_{i}^{\prime} \bar{u}_{j}+\rho^{\prime} \bar{u}_{i} u_{j}^{\prime}+\rho^{\prime} u_{i}^{\prime} u_{j}^{\prime} \\
u_{i}^{\prime}\left(e^{\prime}+p^{\prime}\right)
\end{array}\right) .
\end{gathered}
$$

Neglecting density fluctuations and taking time averages leads to:

$$
\overline{L H S}=\overline{R H S}=\frac{\partial R_{i}}{\partial x_{i}},
$$

where

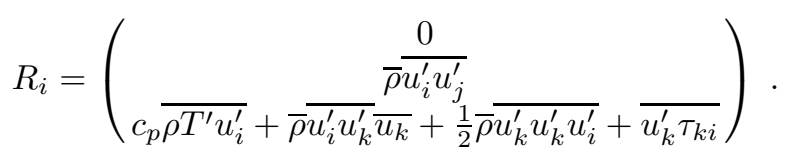

In above equations, the mean-flow quantities are obtained from the separate solution of the RANS equations (11), and the unknown perturbation quantities are obtained from the time-dependent nonlinear disturbance equations (4). The key step in NLAS is to obtain the unknown perturbation terms in $R_{i}$, from the RANS. Once the mean levels and sub-grid sources established at the initial time, by the synthetic reconstruction of turbulence mentioned above, time-dependent computations can then be made to determine the transmitted perturbations about this mean using the above set of disturbance equations (4).

The far-field sound can be computed at the specific observer points using FW-H (Ffowcs Williams-Hawking) acoustic analogy [14], before that, the timedependent surface data was computed and saved in each NLAS step. In the present study, the sound surface is specified as the whole outlet surface, to compute the radiation to far-field, since the outlet is the only surface where the flow induced noise radiated through. While the sound pressure $p^{\prime}(t)$ in time domain obtained from FW-H method, the sound pressure level should be given with FFT tools. 

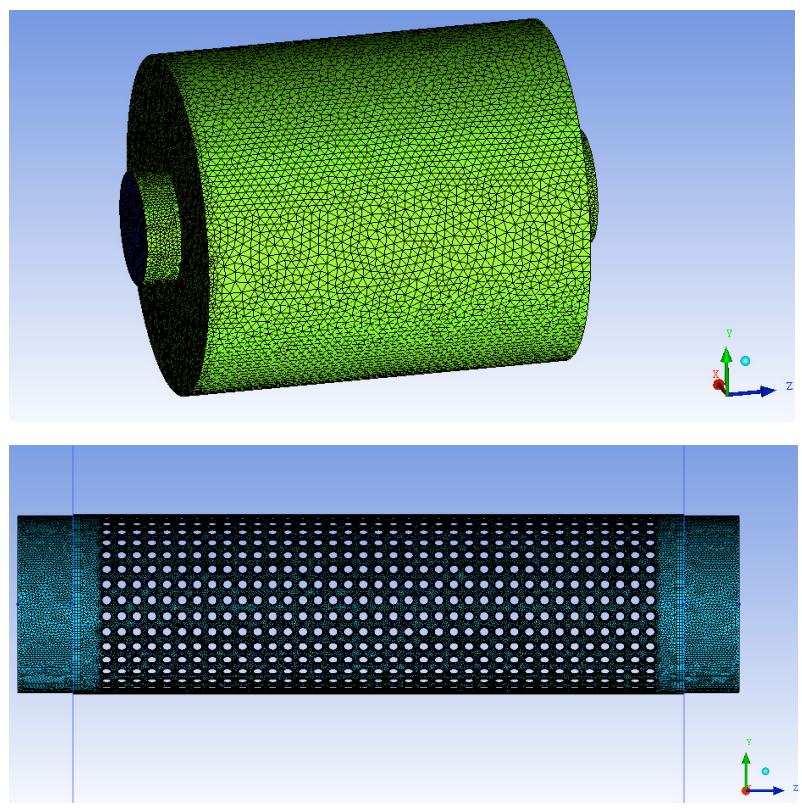

Fig. 1. The grids of (a) muffler-A, exterior view, without perforated wall, (b) muffler$\mathrm{B}$, interior view to show the perforated wall.

\subsection{Physical Model and Boundary Conditions}

The two kinds of mufflers is referred to muffler- $\mathrm{A}$, an expanded chamber without a perforated wall, and muffler-B, with the perforated wall along the duct, which are of the common forms of an acoustic filter. The diameter of the ends of both mufflers is $100 \mathrm{~mm}$, and the total length is $400 \mathrm{~mm}$; the chamber is with diameter of $300 \mathrm{~mm}$ and length of $350 \mathrm{~mm}$. As to muffler-B, the diameter of the holes of the perforated wall is $5 \mathrm{~mm}$, and the porosity ratio of the wall is $25 \%$. The tetrahedral/pyramid/prism mixed-type unstructured grids were generated. The grids around all of the walls were densified, especially around the holes, because the holes is relatively very small. The models' grids are showed in Fig. 1 The number of total elements of muffler-A is 779784 , and that of muffler-B is 10400946, noted that the later is much larger than the former. The flow speed though the muffler is $10 \mathrm{~m} / \mathrm{s}$. For muffler-A, cases of flow speed $5 \mathrm{~m} / \mathrm{s}$ and $8 \mathrm{~m} / \mathrm{s}$ were also simulated.

The standard atmosphere air state, i.e. pressure $101325 \mathrm{~Pa}$ and temperature $298.5 \mathrm{~K}$, was set as the free-stream condition, which was specified at outlet. The inlet turbulent degree is set as 0.01 . The non-slip, adiabatic condition were set at solid wall. 

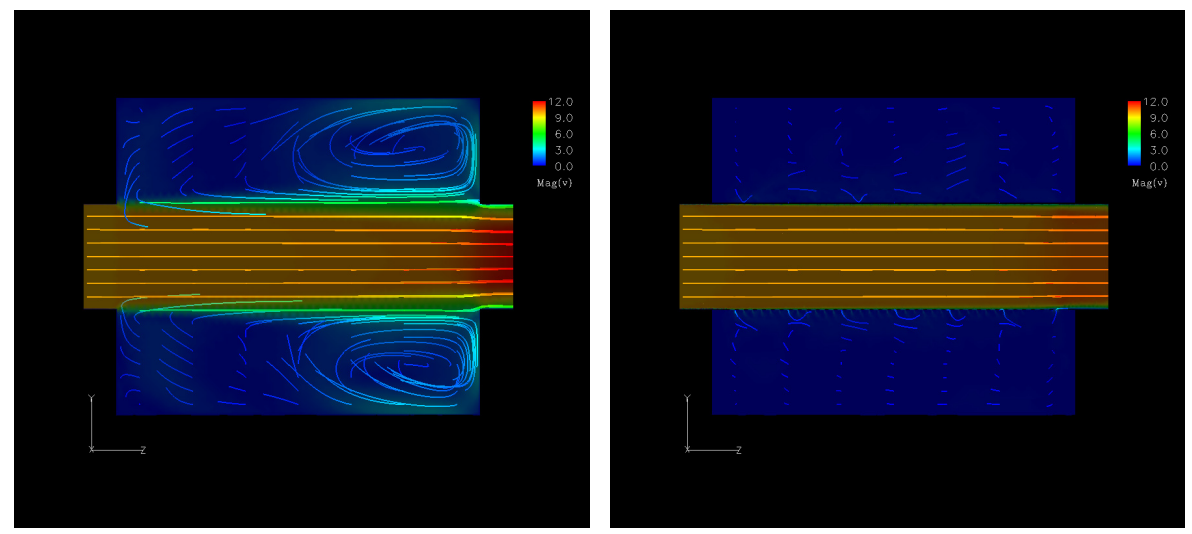

Fig. 2. Streamlines in the middle cross section $(x=0$ plane $)$ in the mufflers, shaded with the velocity magnitude, (a) muffler-A, (b) muffler-B. Note that the seeds to compute the streamlines is at $z=0, \pm 0.05, \pm 0.10, \pm 0.15$, and -0.20 .

\section{Results and Discussions}

In order to distinguish the differences between muffler-A and muffler-B, results are given below with comparisons between them.

\subsection{Mean Flow Physics}

The mean flow is computed by RANS. The mean flow fields of velocity and pressure are showed in Fig. 2 and Fig. 3 , respectively.

In fact, muffler-A is identical to a cavity in a duct, such flow has been studied by many previous works. With respect to muffler-A, the flow from the inlet encounters a backward facing step at first, and a forward facing step before the end of the duct. The flow structures change a lot, that the crosswise flow and refluence happen, influenced by the expanded chamber, or cavity, comparing to the basic pipe flow, which is referred to Poiseuille's flow. On the other hand, to muffler-B, because of the existence of the perforated wall, the influence of the chamber is reduced quite a lot, the flow in the duct remain almost the same as the basic Poiseuille's flow. Fig. 2 reveals these characteristics. It can be seen that flow in the flow field has a discontinuity across the perforated wall in muffler-B, in Fig. 2 and Fig. 3 .

Moreover, the velocity profile, Fig. 4, shows this characteristic difference more clearly. Note that, free shear layer dominates the flow around the steps in mufflerA, and the shear layers is quite thick. Furthermore, in the expanded section there exists refluence and large vortex structures, like the flow in a cavity. However, as to muffler-B, the boundary layer dominate the flow around the perforated wall. It looks like that flow in the chamber and flow in the main duct are separated by the perforated wall, that is, fluid in the chamber retains quiescence almost and the flow in the main duct seems to have no change. 

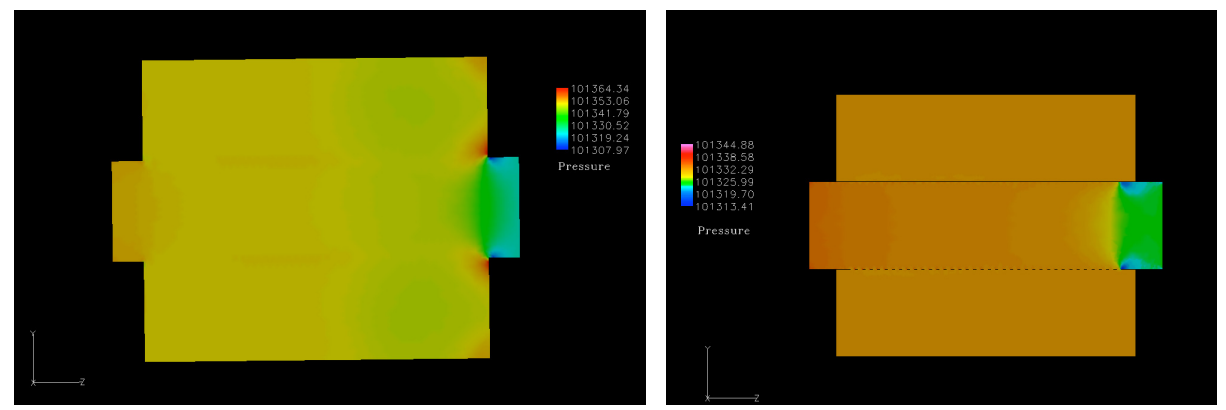

Fig. 3. The mean pressure field in the mufflers (a) muffler-A, (b) muffler-B

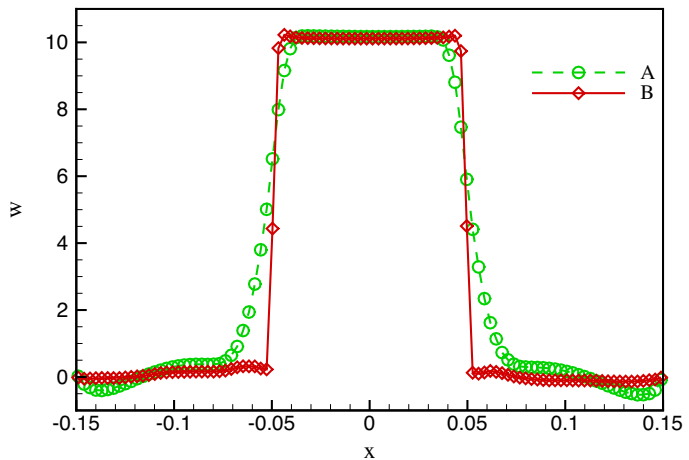

Fig. 4. The velocity profile at the middle cross section $(z=0)$ of muffler-A and muffler-B

\subsection{Sound Field at Near-Field}

The near-field sound field are shown in Fig. 5 represented by the quantity of acoustic pressure, which is defined as $p^{\prime}=p(t)-\bar{p}$, where the transient pressure $p(t)$ is computed by NLAS, and $\bar{p}$ is obtained via RANS. It can be observed that, flow in muffler-A, mainly along the down stream of the backward step, generate remarkable fluctuation. Otherwise, in muffler-B, the magnitude of the pressure fluctuations is much smaller, and there is few visible fluctuated regions.

The noise source mechanism of muffler-A can be considered as the result of the shear layer, where vortexes shed from the backward facing step periodically. Moreover, the shear layer and vortexes impinge into the down corner of the expanded chamber in the down stream. Although, such large and strong vortex shedding does not happen in muffler-B because of the existence of the perforated wall, while small eddies can be observed around the perforated wall, holes and the corner region neighbor to the outlet. 

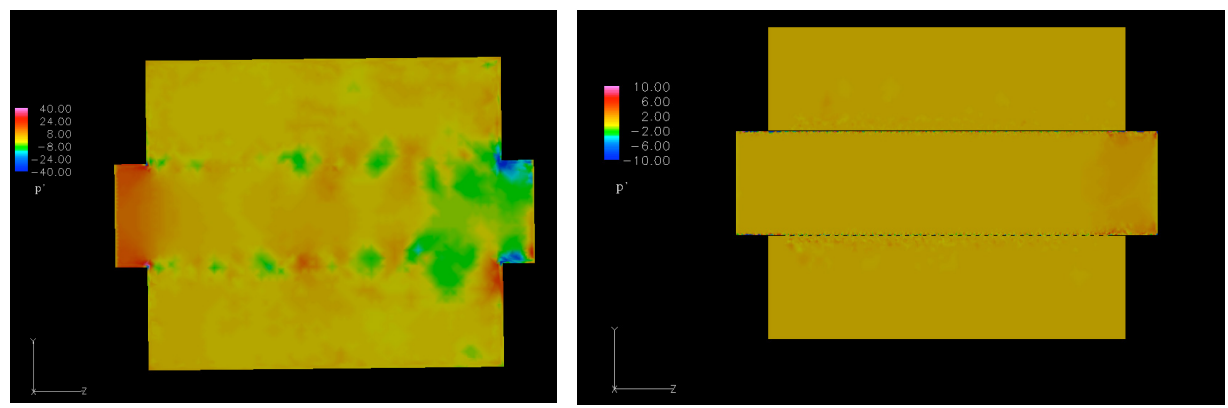

Fig. 5. The acoustic pressure field in the mufflers (a) muffler-A (b) muffler-B

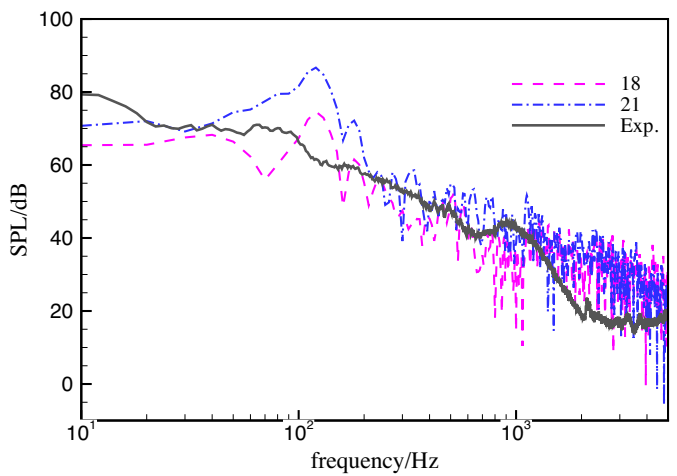

Fig. 6. Sound pressure level spectrum of the pressure fluctuation at the near-field observer point (Exp.) in muffler-B, comparing with the simulation results of the observer points (18 and 21) close to the measuring point, close to the outlet in the axis

With respect to the muffler with perforated wall, muffler-B, the measurement has been done by the authors. Therefore, results were compared to experimental measured, as presented by Fig. 6, where the 18 and 21 represent the observer points located in the axis, close to the outlet in the simulation, near the experimentally measured observer point. It can be considered that the level and spectrum shape results from the simulation are in well agreement with reality.

\subsection{Noise Radiation at Far-Field}

The far-field radiation was computed at observer points at a semi-circle about the center of outlet, in order to compute the directivity of the sound radiation from the outlet. The radius of the semi-circle is $1 \mathrm{~m}$, and the points were arranged with equal circumferential interval of 15 degree. The sound pressure level spectrum is showed by Fig. 7] where $S P L$ represents sound pressure level. It can be seen 

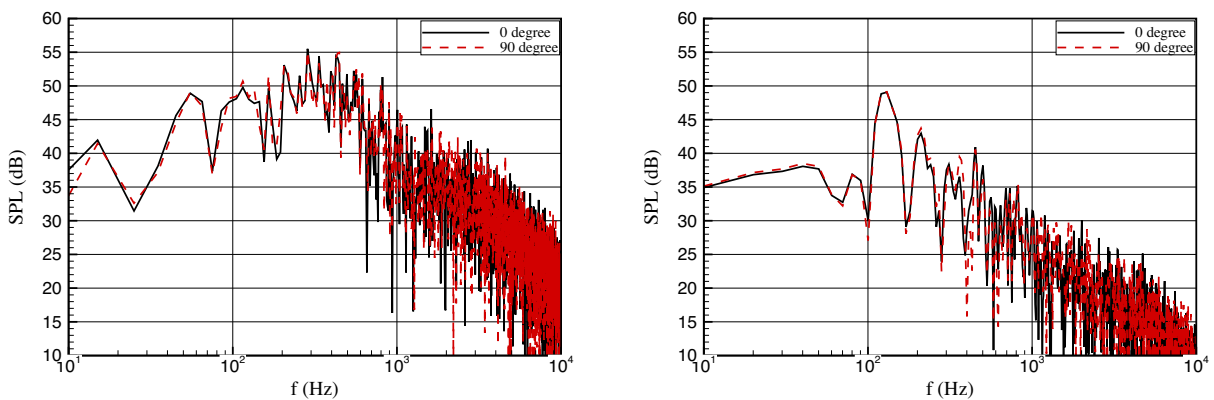

Fig. 7. The sound pressure level spectrum of far-field noise (a) muffler-A, (b) muffler$\mathrm{B}$, where ' 0 degree' indicates axial $(+z)$ direction, and '90 degree' indicates the radial $(+y)$ direction
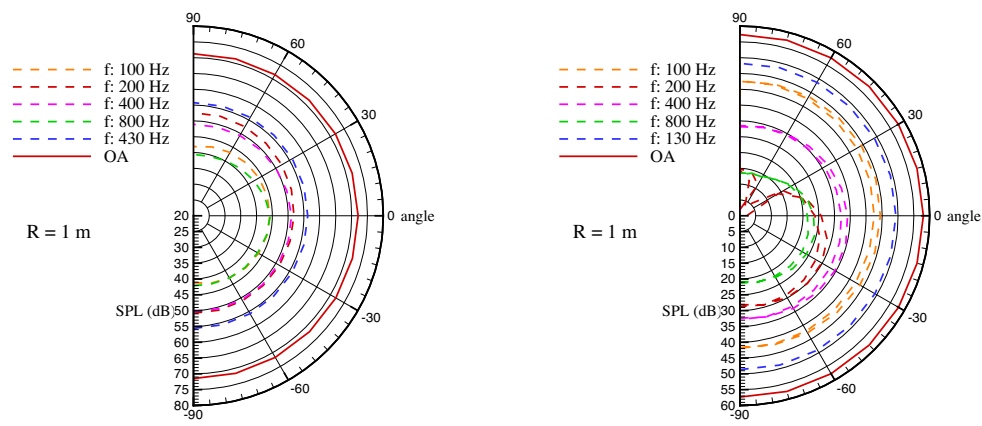

Fig. 8. The directivity of far-field noise, in the specified frequencies and the over-all (OA) value, (a) muffler-A, (b) muffler-B

that, the spectrum of $z$ direction and of $y$ direction coincide mostly. However, the sound pressure level of muffler-A is remarkably larger than that of muffler-B, in most frequency bands.

The far-field noise directivity is shown in Fig. 8, According to Fig. 8, the noise radiated from the mufflers can be regarded as spherical, for the noise level appears equal in almost all directions, except in some single frequencies. Thus, the sound power of the noise radiated from the outlet can be regarded as the sum of the sound power integrated on the unit semi-sphere $(R=1 \mathrm{~m})$, so the sound power level may be calculated as $L_{w}=S P L+10 \lg \left(2 \pi R^{2}\right)=S P L+8.0$ $(\mathrm{dB})$.

Varying the flow speed, the sound pressure level varies. Table. 1 shows results of these cases, with total sound pressure level and sound power level listed. With increase of the flow speed, the noise level increases. The sound power level of muffler-A with flow speed of $10 \mathrm{~m} / \mathrm{s}$ is $79.6 \mathrm{~dB}, 14 \mathrm{~dB}$ larger than that of 
Table 1. The sound power level and sound pressure level of the mufflers

\begin{tabular}{l|llll}
\hline muffler- & A & A & A & B \\
flow speed $(\mathrm{m} / \mathrm{s})$ & 5 & 8 & 10 & 10 \\
\hline$S P L(\mathrm{~dB})$ & 59.6 & 67.9 & 71.7 & 57.7 \\
$L_{w}(\mathrm{~dB})$ & 67.6 & 75.9 & 79.6 & 65.7 \\
\hline
\end{tabular}

muffler-B with the same flow speed, which is qualitatively correct. Because the experiment is difficult, no quantitive data of noise induced purely by flow has been obtained by the authors.

\section{Conclusions}

The flow-induced noise of two kinds of the typical mufflers were investigated, by means of numerical simulation, with a sophisticated approach of computational aeroacoustics. The method has advantages in high accuracy and low computation cost in acoustic simulation. Results show good agreement with the practical measurement in quality and quantity. Several conclusions on the mechanisms and characteristics of the flow physics and flow induced noise of mufflers may be drawn as below.

- The main noise generation mechanism of the mufflers is the shear layer and vortex shedding behind the first cavity corner of the expanded chamber.

- The muffler with the perforated wall produces much smaller flow induced noise than that without the wall in the expanded chamber, in that perforated wall reduces the affection of the chamber and suppress flow fluctuation.

- The radiation from the muffler outlet of the flow induced noise presents spheric directivity, for both mufflers.

Acknowledgments. This work was supported by NSFC (No. 11272325) , Knowledge Innovation of Chinese Academy of Sciences (KJCX2-EW-L02-1) and the Knowledge Innovation Program of Institute of Acoustics, Chinese Academy of Sciences, which are gratefully acknowledged by the authors.

\section{References}

1. Munjal, M., Krishnan, S., Reddy, M.: Flow-Acoustic Performance of Perforated Element Mufflers with Application to Design. Noise Control Eng. J. 40, 159-167 (1993)

2. Broatch, A., Margot, X., Gil, A., Denia, F.: A CFD Approach to the Computation of the Acoustic Response of Exhaust Mufflers. J. Comput. Acoust. 13, 301-316 (2005)

3. Chen, J., Shi, X.: CFD Numerical Simulation of Exhaust Muffler. In: 2011 Seventh International Conference on Computational Intelligence and Security (CIS), pp. 1438-1441. IEEE (2011) 
4. Parsani, M., Ghorbaniasl, G., Lacor, C.: Validation and Application of an HighOrder Spectral Difference Method for Flow Induced Noise Simulation. J. Comput. Acoust. 19, 241-268 (2011)

5. Montenegro, G., Onorati, A., Della Torre, A.: The Prediction of Silencer Acoustical Performances by 1d, 1d-3d and Quasi-3d Non-Linear Approaches. Comput. Fluids 71, 208-223 (2012)

6. Lee, J.W., Jang, G.: Topology Design of Reactive Mufflers for Enhancing Their Acoustic Attenuation Performance and Flow Characteristics Simultaneously. Int. J. Numer. Meth. Eng. 91, 552-570 (2012)

7. Tam, C.K.W.: Computational Aeroacoustics - Issues and Methods. AIAA J. 33, 1788-1796 (1995)

8. Wagner, C., Hüttl, T., Sagaut, P. (eds.): Large Eddy Simulation for Acoustics. Cambridge University Press (2007)

9. Batten, P., Goldberg, U., Chakravarthy, S.: Reconstructed Sub-Grid Methods for Acoustics Predictions at All Reynolds Numbers. AIAA-2002-2511 (2002)

10. Batten, P., Ribaldone, E., Casella, M., Chakravarthy, S.: Towards a Generalized Non-Linear Acoustics Solver. AIAA-2004-3001 (2004)

11. Karypis, G.: METIS - Family of Multilevel Partitioning Algorithms (2008), http://glaros.dtc.umn.edu/gkhome/views/metis

12. Weiss, J.M., Smith, W.A.: Preconditioning Applied to Variable and Constant Density Flows. AIAA J. 33, 2050-2057 (1995)

13. Morris, P.J., Long, L.N., Bangalore, A., Wang, Q.: A Parallel Three-Dimensional Computational Aeroacoustics Method Using Nonlinear Disturbance Equations. J. Comput. Phys. 133, 56-74 (1997)

14. Ffowcs Williams, J.E., Hawkings, D.L.: Sound Generation by Turbulence and Surfaces in Arbitrary Motion. Philos. T. Roy. Soc. A 264, 321-342 (1969) 